University of Nebraska - Lincoln

DigitalCommons@University of Nebraska - Lincoln

\title{
Conjugate Heat Transfer From a Two- Layer Substrate Model of a Convectively Cooled Circuit Board
}

\author{
S. A. Harman \\ University of Nebraska-Lincoln \\ Kevin D. Cole \\ University of Nebraska-Lincoln, kcole1@unl.edu
}

Follow this and additional works at: https://digitalcommons.unl.edu/mechengfacpub

Part of the Mechanical Engineering Commons

\footnotetext{
Harman, S. A. and Cole, Kevin D., "Conjugate Heat Transfer From a Two- Layer Substrate Model of a Convectively Cooled Circuit Board" (2001). Mechanical \& Materials Engineering Faculty Publications. 46. https://digitalcommons.unl.edu/mechengfacpub/46
}

This Article is brought to you for free and open access by the Mechanical \& Materials Engineering, Department of at DigitalCommons@University of Nebraska - Lincoln. It has been accepted for inclusion in Mechanical \& Materials Engineering Faculty Publications by an authorized administrator of DigitalCommons@University of Nebraska Lincoln. 


\section{Journal of}

Electronic

Packaging

\section{Technical Brief}

\section{Conjugate Heat Transfer From a Two- Layer Substrate Model of a Convectively Cooled Circuit Board}

S. A. Harman and K. D. Cole

Mechanical Engineering Dept., University of Nebraska, Lincoln, NE 68588

\section{Introduction}

This note presents the results of a study of a simple model of an air-cooled electronic device mounted on a circuit board. The model includes a flush-mounted heater to represent the electronic device, a two-layer substrate to represent the circuit board, and a steady shear flow to represent the cooling fluid.

Ortega [1] provides a good review of the literature and a comprehensive introduction to conjugate methods applied to electronic cooling, emphasizing simple shear flows and single-layer substrates to illustrate basic principles. Ortega also describes twolayer effects by including infrared thermographs of a convectively cooled glass/epoxy circuit board with copper traces, which produce significant anisotropy, with larger in-plane relative to out-ofplane conductivity in the circuit board.

There have been few conjugate studies with multiple conduction layers. Zebib and Wo [2] simulated a protruding electronic module on a three-layer circuit board, but the geometry was fixed and only three fluid velocities were used. Gorobets [3] analyzed a fin with a low-conductivity coating to simulate the effects of fin fouling. Chen et al. [4] developed a solution that included solid, liquid, and gas phases to simulate laminar condensation on a fin. In a series of papers Lee and co-workers (Palisoc and Lee [5], Lee et al. $[6,7,8])$ studied several different substrates with up to five layers, but these studies approximated the convective cooling by a spatially-uniform heat transfer coefficient.

This note builds upon the work of one of us (Cole [9]) for a single-layer substrate, in which results were presented for various values of the dimensionless substrate thickness and the conjugate Peclet number, $\left(k_{f} / k_{s}\right) \mathrm{Pe}^{1 / 3}$. The present study adds a second layer to the substrate described by a single additional parameter that combines the thickness and conductivity of the added layer.

\section{Analysis}

The circuit board is modeled as a two-layer substrate of infinite lateral extent with a flush-mounted heater of streamwise length $2 \mathrm{a}$ generating heat uniformly. Refer to Fig. 1. The top layer of the substrate is thin and highly conductive and treated as a lumped solid in the $y$-direction. The bottom of the substrate is insulated. The fluid flow is a spatially invariant shear flow with velocity

Contributed by the Electrical and Electronic Packaging Division of THE AMERICAN SOCIETY OF MECHANICAL ENGINEERS. Manuscript received by the EEPD March 31, 2000. Associate Editor: R. Schmidt. gradient $\beta$. Thermal properties are constant (small temperature rise assumed). Radiation, natural convection, and viscous dissipation are neglected. The energy equations for the fluid flow and the two substrate layers are given below.

$$
\begin{gathered}
\text { fluid : } \quad \beta y_{f} \frac{\partial T_{f}}{\partial x}=\frac{\partial^{2} T_{f}}{\partial y_{f}^{2}} ; 0<y_{f}<\infty \\
\text { solid 1: } \quad k_{s 1} D_{1} \frac{\partial^{2} T_{s 1}}{\partial x^{2}}=\left.k_{f} \frac{\partial T_{f}}{\partial y_{f}}\right|_{y_{f}=0}+\left.k_{s 2} \frac{\partial T_{s 2}}{\partial y_{s 2}}\right|_{y_{s 2}=0}+q_{0} p(x) \\
\text { solid 2: } \frac{\partial^{2} T_{s 2}}{\partial y_{s 2}^{2}}+\frac{\partial^{2} T_{s 2}}{\partial x^{2}}=0 ; \quad 0<y_{s 2}<D_{2}
\end{gathered}
$$

Coordinate $x$ is the streamwise direction with range $(-\infty<x$ $<\infty)$ and subscripts ()$_{f},()_{s 1}$, and ()$_{s 2}$ refer to the fluid, solid 1, and solid 2, respectively. Function $q_{0}$ is the heat flux introduced by the heater and $p(x)$ is the top-hat function defined as unity on $(-a<x<a)$ and zero elsewhere.

The temperature and heat flux at the interface are obtained by solving the above energy equations in the fluid and both solids separately with continuity of temperature at the fluid-solid and solid-solid boundaries. Nondimensionalization of the governing equations yields three dimensionless governing parameters defined as follows: conjugate Peclet number $\Lambda$ $=\left(k_{f} / k_{s 2}\right)\left(\beta a^{2} / k_{f}\right)^{1 / 3} ; \quad D_{2}^{+}=D_{2} / a ; \quad$ and, $\quad D_{1}^{+} K=\left(D_{1} / a\right)$ $\times\left(k_{s 1} / k_{s 2}\right)$. Solutions are obtained through a combined numeri$\mathrm{cal}$ and analytical approach. The nondimensional governing equations are Fourier transformed in the $x$-direction reducing them to ordinary differential equations with analytical solutions. The realspace solutions are then given as integrals with the inverse Fourier transform, which must be evaluated numerically. For the details of the analysis see Harman [10].

\section{Results}

Temperature Distribution. Figure 2 shows the normalized temperature at the fluid-solid interface versus $x$ for $\Lambda=1, D_{2}^{+}$ $=100$, and for various $D_{1}^{+} K$. Increasing $D_{1}^{+} K$ increases the ability of the top solid layer to carry heat, which boosts the rate of heat flow into the solid and distributes it farther upstream and downstream before it enters the fluid. Consequently, as $D_{1}^{+} K$ increases the temperature peak becomes broader, lower, and more symmetric. Similar features are present in the heat flux field (not shown).

As $D_{1}^{+} K$ is increased and the top solid layer carries more heat, the bottom solid layer carries less heat, and the temperature field spreads out over lengths much greater than the heater length. If $D_{2}^{+}$is small compared to $D_{1}^{+} K$, the temperature distribution across the thickness of the bottom layer can be neglected and the entire solid treated as a single lumped-capacitance layer with effective thickness $D_{\text {eff }}^{+}=\left(D_{1}^{+} K+D_{2}^{+}\right)$; this is called the lumped-solid limit. This limit is also approached if the convective flow velocity is small (small conjugate Peclet number) because as convection decreases the shape of the temperature field becomes broader and 


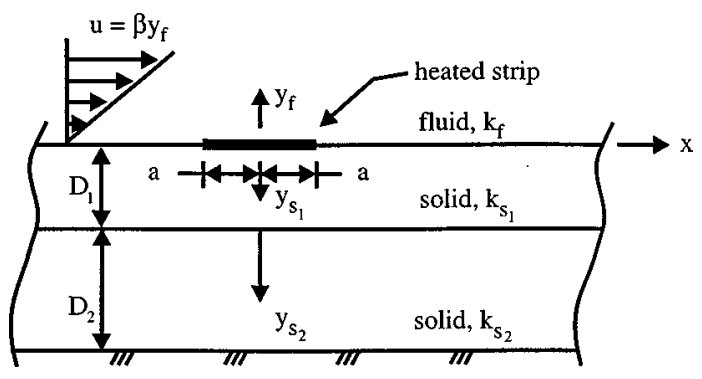

Fig. 1 Conjugate heat transfer geometry

more symmetric; however the peak temperature increases. In the limit $\Lambda \rightarrow 0$ the peak temperature increases without limit; this is a consequence of the two-dimensional geometry.

Spatial Average Temperature. Figure 3 shows the spatial average modified temperature on the heated region, $\Lambda \overline{T^{+}}=\Lambda(\bar{T}$ $\left.-T_{\infty}\right) k_{s 2} /\left(q_{0} a\right)$, versus $\Lambda / D_{\text {eff }}^{+}$at $D_{2}^{+}=100$ and various $D_{1}^{+} K$. This modified temperature is used to preserve the conjugate Peclet number $\Lambda$ as a basic parameter and to show the limiting behavior at large and small values of $\Lambda$. The convergence of the curves for small $\Lambda$ is the lumped-solid limit. The large- $\Lambda$ asymptote $\Lambda \overline{T^{+}}$

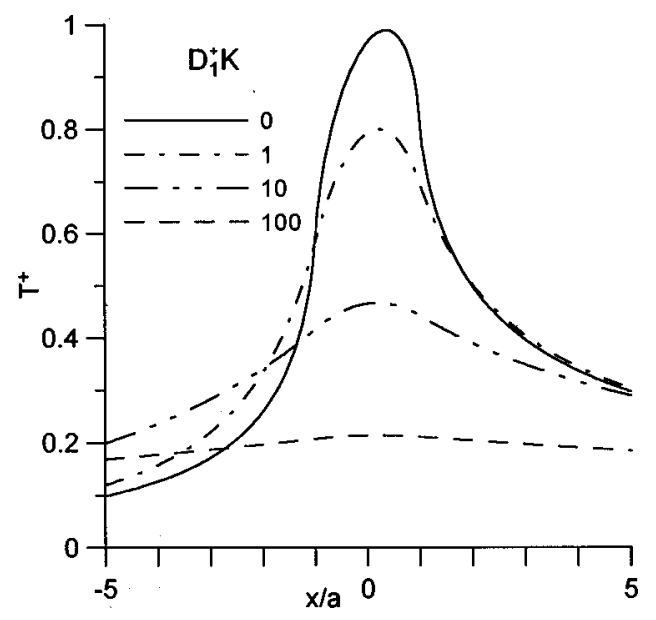

Fig. 2 Dimensionless heat flux to the fluid at $\Lambda=1, D_{2}^{+}=100$, and various $D_{1}^{+} K$

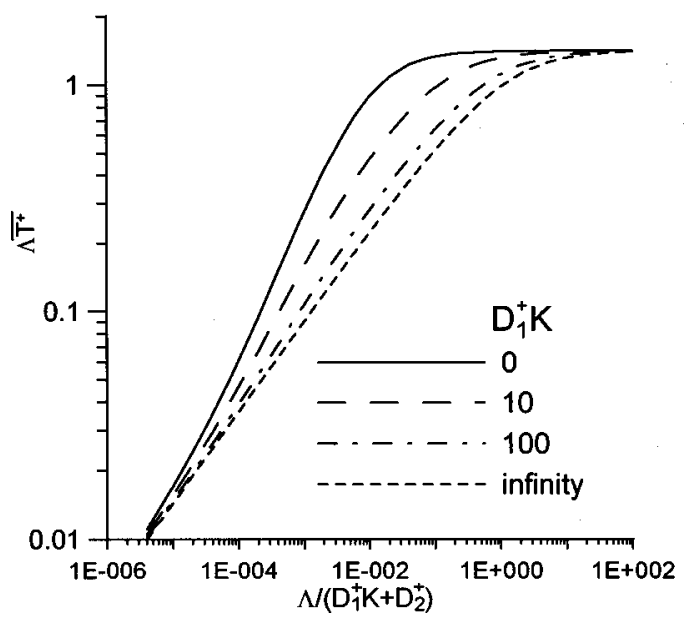

Fig. 3 Modified spatial average temperature versus $\Lambda / D_{\text {eff }}^{+}$for $D_{2}^{+}=100$, and various $D_{1}^{+} K$

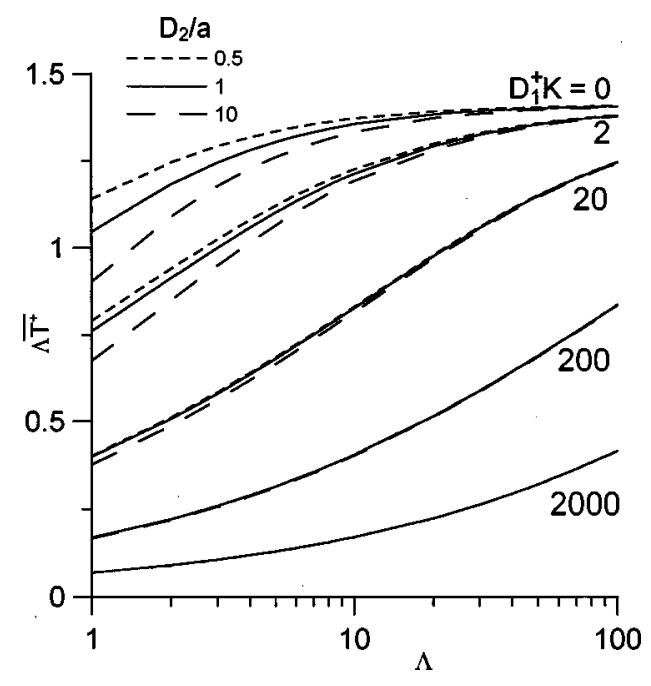

Fig. 4 Modified spatial average temperature versus $\Lambda$ for various $D_{2}^{+}$, and $D_{1}^{+} K$

$=1.44$ is associated with convection-dominated heat transfer. In this region the temperature varies inversely with the conjugate Peclet number; that is, $\overline{T^{+}} \sim 1 / \Lambda$.

The range $1<\Lambda<100$ represents a large number of real-world flow geometries, including air-cooled circuit boards. Figure 4 shows the spatial average modified temperature in this range of $\Lambda$ for various values of $D_{1}^{+} K$ and $D_{2}^{+}$. The impact of $D_{2}^{+}$diminishes as $\Lambda$ increases since increasing convection begins to mask the effect of the bottom solid layer. The impact of $D_{2}^{+}$also diminishes at greater values of $D_{1}^{+} K$; for $D_{1}^{+} K>20$ variation in $D_{2}^{+}$has almost no effect on the spatial average temperature.

Table $1 \overline{N_{u}^{*}}$ for various $\Lambda, D_{1}^{+}$, and $D_{2}^{+}$

\begin{tabular}{|l|l|l|l|l|l|l|l|l|l|l||}
\hline \hline \multicolumn{1}{|c|}{} & \multicolumn{7}{|c||}{$D_{1}^{+} K$} \\
\hline$\Lambda$ & $D_{2}^{+}$ & $10^{3}$ & $10^{2}$ & $10^{1}$ & $10^{.5}$ & $10^{0}$ & $10^{-.5}$ & $10^{-1}$ & $10^{-2}$ & 0 \\
\hline 1 & .5 & .3290 & .5071 & .7392 & .8711 & .9914 & 1.078 & 1.126 & 1.155 & 1.158 \\
\hline 1 & 1 & .3290 & .5068 & .7348 & .8590 & .9651 & 1.037 & 1.074 & 1.096 & 1.099 \\
\hline 1 & 10 & .3286 & .5033 & .7172 & .8293 & .9237 & .9880 & 1.022 & 1.044 & 1.047 \\
\hline 2 & .5 & .7530 & 1.146 & 1.649 & 1.924 & 2.170 & 2.344 & 2.439 & 2.496 & 2.503 \\
\hline 2 & 1 & .7530 & 1.146 & 1.640 & 1.901 & 2.123 & 2.272 & 2.351 & 2.400 & 2.407 \\
\hline 2 & 10 & .7520 & 1.139 & 1.612 & 1.856 & 2.061 & 2.200 & 2.276 & 2.324 & 2.331 \\
\hline 5 & .5 & 2.236 & 3.318 & 4.715 & 5.424 & 6.022 & 6.402 & 6.630 & 6.767 & 6.789 \\
\hline 5 & 1 & 2.236 & 3.315 & 4.695 & 5.375 & 5.932 & 6.293 & 6.486 & 6.613 & 6.633 \\
\hline 5 & 10 & 2.234 & 3.302 & 4.640 & 5.302 & 5.839 & 6.189 & 6.377 & 6.504 & 6.524 \\
\hline 10 & .5 & 5.075 & 7.442 & 10.35 & 11.75 & 12.84 & 13.53 & 13.89 & 14.15 & 14.19 \\
\hline 10 & 1 & 5.075 & 7.380 & 10.32 & 11.67 & 12.72 & 13.36 & 13.71 & 13.95 & 13.99 \\
\hline 10 & 10 & 5.072 & 7.417 & 10.25 & 11.58 & 12.60 & 13.23 & 13.58 & 13.82 & 13.86 \\
\hline 50 & .5 & 33.20 & 47.42 & 62.03 & 67.4 & 70.83 & 72.85 & 73.99 & 74.91 & 75.10 \\
\hline 50 & 1 & 33.20 & 47.90 & 61.92 & 67.23 & 70.61 & 72.61 & 73.74 & 74.64 & 74.84 \\
\hline 50 & 10 & 33.18 & 47.35 & 61.80 & 67.09 & 70.46 & 72.45 & 73.57 & 74.48 & 74.67 \\
\hline 100 & .5 & 74.48 & 104.1 & 131.4 & 139.9 & 145.2 & 148.3 & 150.1 & 151.6 & 151.9 \\
\hline 100 & 1 & 74.47 & 104.0 & 131.2 & 139.7 & 144.9 & 148.0 & 149.8 & 151.3 & 151.6 \\
\hline 100 & 10 & 74.45 & 104.0 & 131.1 & 139.5 & 144.8 & 147.9 & 149.6 & 151.1 & 151.4 \\
\hline \hline
\end{tabular}


The values of $D_{2}^{+}$shown in Fig. 4 are representative of the entire range of behaviors $\left(0<D_{2}^{+}<\infty\right)$ since the $D_{2}^{+}=0.5$ curves shown are nearly identical to the lumped-solid solution, and the $D_{2}^{+}=10$ curves shown are very close to the semi-infinite solution $\left(D_{2}^{+} \rightarrow \infty\right)$.

Correlations of Results. In the range $1<\Lambda<100$ and where the lumped-solid approximation applies $\left(D_{2}^{+}<0.5\right)$, the following correlations are accurate within 5 percent:

for $\Lambda / D_{\text {eff }}^{+}>.55$;

$$
\Lambda \bar{T}^{+}=1.44-0.4673\left(\frac{\Lambda}{D_{\text {eff }}^{+}}\right)^{-2 / 3}-.06363\left(\frac{\Lambda}{D_{\text {eff }}^{+}}\right)^{-4 / 3}
$$

for $\Lambda / D_{\text {eff }}^{+}<.55$;

$$
\Lambda \bar{T}^{+}=1.4619\left(\frac{\Lambda}{D_{\text {eff }}^{+}}\right)^{2 / 5}-.2895\left(\frac{\Lambda}{D_{\text {eff }}^{+}}\right)^{4 / 5}-.1695\left(\frac{\Lambda}{D_{\text {eff }}^{+}}\right)^{6 / 5}
$$

for $\Lambda / D_{\text {eff }}^{+}>1.2$;

$$
\frac{\overline{N_{u}^{*}}}{\Lambda}=1.52-.728\left(\frac{\Lambda}{D_{\text {eff }}^{+}}\right)^{-3 / 4}+.3363\left(\frac{\Lambda}{D_{\text {eff }}^{+}}\right)^{-3 / 2}
$$

for $\Lambda / D_{\text {eff }}^{+}<1.2$;

$$
\frac{\overline{N_{u}^{*}}}{\Lambda}=1.3819\left(\frac{\Lambda}{D_{\text {eff }}^{+}}\right)^{1 / 5}-0.1627\left(\frac{\Lambda}{D_{\text {eff }}^{+}}\right)^{2 / 5}-0.1068\left(\frac{\Lambda}{D_{\text {eff }}^{+}} K\right)^{3 / 5}
$$

where $\overline{N_{u}^{*}}=\bar{h} a / k_{s 2}$, called the modified spatial average Nusselt number (actually a Biot number), where $\bar{h}$ is the spatial-average heat transfer coefficient on the heated region.

For larger values of $D_{2}^{+}$correlations were found to be impractical, so a table of values has instead been provided from which values can be interpolated with 95 percent accuracy. Values given in Table 1 for $\overline{N_{u}^{*}}$ can be linearly interpolated directly with respect to $D_{2}^{+}$. The logarithm of $\overline{N_{u}^{*}}$ can be linearly interpolated with respect to the logarithm of $D_{1}^{+} K$. Finally, with respect to variation in $\Lambda$, the ratio $\overline{N_{u}^{*}} / \Lambda$ can be linearly interpolated with the logarithm of $\Lambda$. The low convection limit applies for $D_{1}^{+} K>10^{3}$ and table values can be extrapolated as follows: $\overline{N_{u}^{*}}=\overline{N_{u}^{*}}(\Lambda$
=100) $\Lambda^{1 / 5} /(100)^{1 / 5}$. The $D_{2}^{+}=10$ case adequately represents results for $10<D_{2}^{+}<\infty$ (with at least 98 percent accuracy).

\section{Conclusion}

In this paper results of a conjugate analysis applied to the electronic cooling problem are presented. The analysis is an extension of earlier work with a single-layer substrate by adding a second solid layer to represent the surface traces on a circuit board. The addition of a lumped-solid second layer to the substrate adds a single dimensionless parameter to the problem, $D_{1}^{+} K$. Numerical results for the two-layer substrate model are presented over a wide range of flow parameter $\Lambda$, bottom layer thickness $D_{2}^{+}$, and toplayer parameter $D_{1}^{+} K$. Design correlations and a table of values are given spatial average (modified) Nusselt number to characterize thermal behaviors for values of the governing parameters typically encountered in electronic cooling applications.

\section{References}

[1] Ortega, A., 1996, "Conjugate Heat Transfer in Forced Air Cooling of Electronic Components," Air Cooling Technology for Electronic Equipment, S. Kim and S. Lee, eds., CRC Press, New York, pp. 107-114.

[2] Zebib, A., and Wo, Y. K., 1989, "A two-dimensional conjugate heat transfer model for forced air cooling of an electronic device,' ASME J. Electron. Packag., 111, pp. 41-45.

[3] Gorobets, V. G., 1995, "Local and global heat transfer performance of a plate fin with a low thermal conductivity coating," Heat Transfer Research, 26 , Nos. 3-8, pp. 445-461.

[4] Chen, H., Lan, Z., and Wang, T. I., 1994, "'Study of conjugate conductionlaminar film condensation for a vertical plate fin,' Int. J. Heat Mass Transf., 37, No. 16, pp. 2592-2597.

[5] Palisoc, A., and Lee, C. C., 1988, “Thermal properties of the multilayer infinite plate structure,' J. Appl. Phys., 64, No. 64, pp. 410-415.

[6] Lee, C. C., Palisoc, A., and Min, Y., 1989, "Thermal Analysis of Integrated Circuit Devices and Packages,' IEEE Trans. Compon., Hybrids, Manuf. Technol., 12, No. 4, pp. 701-709.

[7] Lee, C. C., Min, Y., and Palisoc, A., 1989, “'A General Integration Algorithm for the Inverse Fourier Transform of Four-Layer Infinite Plate Structures," IEEE Trans. Compon., Hybrids, Manuf. Technol., 12, No. 4, pp. 710-716.

[8] Lee, C. C., and Min, Y., 1991, "Thermal Modeling of Semiconductor Devices and Packages using Analytical Methods,' National Electronic Packaging and Production Conference, Anaheim, CA, Vol. 1, pp. 147-160.

[9] Cole, K. D., 1997, “Conjugate heat transfer from a small heated strip,' Int. J. Heat Mass Transf., 40, No. 11, pp. 2709-2719.

[10] Harman, S. A., 1998, "Conjugate heat transfer: the effects of axial conduction and the two component wall,'" M. S. thesis, University of Nebraska, Lincoln, NE. 